\title{
Methods of evaluation of organizational decisions
}

\author{
Pavel Oleinik ${ }^{1}$, and Nadezhda Cherednichenko ${ }^{1,}{ }^{*}$ \\ ${ }^{1}$ Moscow State University of Civil Engineering, Yaroslavskoe shosse, 26, Moscow, 129337, Russia
}

\begin{abstract}
The article considers the levels of organizational and technological documentation, solution of which is subject to evaluation (construction projects organization, labor production projects, technologic maps, particular sections of documents and solution of local objectives). Groups and a list of applied assessment indicators are also given. The approaches of private and complex evaluation of organizational solutions are revealed - methods of criteria estimation (average weighted, additive, multiplicative, generalizing) methods for modeling indicators (continuity, combination, rhythmicity, uniformity, intensity of production labor), multiple correlation methods (polynomials of the $1^{\text {st }}$ and $\mathrm{n}^{\text {th }}$ degree). At the same time, the conditions for the expedient application of such methods with appropriate recommendations and explanations are described.
\end{abstract}

\section{Introduction}

Solutions for the organization of construction production are designed to ensure the rhythmic commissioning of production facilities and fixed assets according to the deadlines on the basis of continuous and uniform loading of the production capacities of construction organizations, determined by the availability of labor resources equipped with means and objects of labor $[1,2,3]$.

Formation and selection of organizational solutions is carried out in the construction organization projects (COP), demolition (disassembling) organization projects (DOP), labor production projects (LPP), technological maps (TM), as well as in the documentation for the organization of labor when preparing a construction organization for the construction of all facilities provided for in the production program [4].

A large number of various organizations (customer services and design organizations, construction, installation and project-technological organizations, manufacturers and others) participate in the implementation of organizational decisions. In this regard, the basic requirements for the organization of construction production are reduced not only to the development of rational organizational solutions aimed at achieving high technical and economic indicators of construction, but also to ensure the unity and sustainability of such solutions during the entire investment process of creating an object $[5,6,7]$.

\footnotetext{
Corresponding author: CherednichenkoND@mgsu.ru
} 


\section{Methodology}

Thus, when designing the organization of construction and production of construction and installation works, as a rule, it is always necessary to choose the best solutions based on multivariance, the implementation of which will ensure the achievement of certain high indicators. For this purpose, various optimality criteria and corresponding constraints are used, allowing one to obtain quantitative estimates of solutions with subsequent comparison between them $[8,9]$.

Such assessment is subject to:

Level I. Project and organizational and technological documents in general (COP, DOP, LPP, TM);

Level II. Separate sections of the documents (calendar construction plan of the facility, schedule of reconstruction of the object, schedule of demolition (dismantling) of the object, calendar construction plan for the preparatory period, schedule of works on the object, calendar plan of construction of objects of the annual (two-year) program of the construction organization, general site construction master plan, object construction master plan, organization of material and technical support, organization of production life, etc.);

Level III. Solutions to local problems (choice and distribution of labor and technical resources, placement of assembly cranes and mechanisms, organization of warehousing facilities construction of internal roads, energy and water provision, organization of supply of material and technical resources, etc.).

The indicators used for the estimates can be divided into three groups (Table 1).

Table 1. Distribution of indicators by solution levels

\begin{tabular}{|c|c|c|c|}
\hline \multirow{2}{*}{$\begin{array}{c}\text { Assessment } \\
\text { Indicator } \\
\text { group }\end{array}$} & \multicolumn{3}{|c|}{ Recommended indicators of evaluation } \\
\cline { 2 - 4 } & Level I & Level II & Level III \\
\hline $1^{\text {st }}$ group & + & + & \\
\hline $2^{\text {nd }}$ group & + & + & \\
\hline $3^{\text {rd }}$ group & & + & + \\
\hline
\end{tabular}

The first group includes such indicators as the total duration of construction, the duration of the preparatory period, the duration of installation of technological equipment, the prime cost of construction, the prime cost of construction and installation works, labor costs, level of mechanization, unit labor costs, efficiency of workers, etc. These indicators reflect the influence of the whole set of factors of the construction system, including the factors of the organization of construction production, technology and mechanization of construction and installation works, and, ultimately, characterize the development of the entire sphere of construction $[10,11,12]$.

The second group contains indicators of the implementation of flow methods, level of threading, implementation of the nodal method, increasing of mobility, etc. However, these indicators are fixing only the degree of introduction of progressive forms and methods of 
organization of construction and cannot serve as an objective general assessment of organizational decisions.

In relation to a specific object, the third group of indicators is the most diverse and perfect, in terms of completeness of accounting for the development of the construction production: continuity, combination, rhythm, uniformity, intensity of construction of buildings and structures, as well as derivatives of these indicators (a simple front of work, specific duration of work, parallelism of processes, etc.). [13, 14, 15].

The approaches of complex evaluation of solutions are divided into three types - methods of criteria assessment, methods of modeling indicators and methods of multiple correlation.

The first group of indicators is used to apply the methods of criteria assessment. They can be both private and integral. Different methods can be used to combine particular indicators into integral ones (definition of weighted average indicator, application of the additive optimality criterion, use of multiplicative generalized criterion of optimality).

\section{Main part}

The integral indicator $(F)$ in case of the weighted average method is defined as:

$$
F=\frac{\sum_{i=1}^{m} K_{i} \varphi_{i}}{\sum_{i=1}^{m} K_{i}}
$$

where $K_{i}$ is significance coefficient $i^{\text {th }}$ criteria;

$\varphi_{i}$ is value of $i^{\text {th }}$ criteria;

$m$ is number of $i^{\text {th }}$ criteria.

The use of the additive criterion of optimality is appropriate if there is a measurement of all the considered particular indicators on one scale and provided that all the particular indicators are equally important. The additive optimality criterion formula is:

$$
A_{j}=\max \frac{1}{n} \sum_{i=0}^{n} \alpha_{i j}
$$

where $\mathrm{A}_{j}$ is an additive optimality criterion;

$\alpha_{i j}$ is a value of the $j^{\text {th }}$ index for the $i^{\text {th }}$ variant;

$\mathrm{n}$ is number of indicators.

The use of the multiplicative generalized criterion of optimality is carried out in case if the total level of relative decline of one or more private indicators does not exceed the total level of the relative increase of the other indicators. The value of this indicator is determined by simple multiplying the particular indicators by the formula:

$$
B_{i}=\prod_{j=1}^{n} b_{i j}
$$

where $\mathrm{B}_{i}$ is a multiplicative generalized criterion of optimality;

$b_{i j}$ are the values of $j^{\text {th }}$ поindex for $i^{\text {th }}$ variant.

When applying modeling methods to assess organizational decisions, the indicators of continuity, alignment, rhythm, uniformity and intensity of work are used. However, the relative measures of such indicators have a significant spread, which does not allow to clearly assess the level of decisions of the organization of construction process. In this regard, some authors propose to take the uniformity of work as the main indicator of such a level of assessment, others suggest to consider the combination of works and so on. How- 
ever, it should be kept in mind that the evaluation of decisions based on one indicator does not mean that the other criteria are going to be high.

The indicator of work continuity $\left(u_{1}\right)$ is determined by the ratio of the time of continuous execution of works at the facility $\left(t_{l}\right)$ to their total duration $(T)$, as:

$$
u_{1}=\frac{t_{1}}{T}
$$

For resources, this continuity indicator shows the extent to which resources are used over time. When applying the indicator of continuity of work, the entire duration of the construction of the object or the annual (two-year) work program $T$ should be divided into equal intervals of time, within which the continuity of work is determined $t_{l}$.

The indicator of work combination $\left(u_{2}\right)$ is characterized by the ratio of time of processes parallel execution $\left(t_{2}\right)$ to their total duration.

$$
u_{2}=\frac{t_{2}}{T}
$$

For resources, the indicator demonstrates the level of provision of field of operations and characterizes its degree of density in the calendar plan. It should be kept in mind that for specialized flows, the field of operations should correspond to the specialty of resources, which will ensure the maximum combination of work with the coordination of the specialization of brigades.

The indicator of rhythm $\left(u_{3}\right)$ determines the degree of over time repeatability of construction products (grabs, site, facility) for a suitable flow or work program and is calculated, for example, for an object flow as:

$$
u_{3}=1-\sigma_{1} / \alpha \cdot n
$$

where: $\sigma_{l}$ - standard deviation of the object flow unit fulfilment;

$\alpha$ is the number of threads;

$n$ is the number of captures of the specialized thread.

$\sigma_{l}$ parameter is determined by the following sum:

$$
\sigma_{l}=\sigma_{2}+\sum_{i=1}^{m} \sigma_{3, i}
$$

where: $\sigma_{2}$ is the standard deviation of the rhythms of the specialized object flow from the rhythm of the object flow, which is defined as:

$$
\sigma_{2}= \pm \frac{\sqrt{\Sigma\left|R_{i}-R\right|^{2}}}{a}
$$

$\sigma_{3, i}-$ the standard deviation of the duration of the works on the grips of their rhythms is defined as:

$$
\sigma_{3, i}= \pm \frac{\sqrt{\sum\left(t_{i}-R_{i}\right)^{2}}}{n}
$$

$R$ is rhythm of object flow in the form of:

$$
R=\frac{\sum_{i} \sum_{j} t_{i j}}{\alpha n}
$$

$R_{i}$ is rhythm of the specialized thread in the form of: 


$$
R_{i}=\frac{\sum_{j} t_{i j}}{n}
$$

An indicator of the uniformity of the production works $\left(u_{4}\right)$ is determined by the ratio of the amount of work performed with constant intensity $\left(V_{l}\right)$ to total volume of the workflow $(V)$, i.e.

$$
u_{4}=\frac{V_{1}}{V}
$$

The figure in the calendar plan characterizes the constancy of intensity of the processes in time, and shows lack or redundancy for resources.

In calculations, $V_{1}$ parameter can be represented as the difference between the volume of work and the total value of its deviation in all time intervals.

The indicator of work production intensity $\left(u_{5}\right)$ is defined in depending on the continuity, alignment and rhythm in the workflow. Each value of the intensity indicator corresponds to their specific values of these indicators. In general, $u_{5}$ indicator is determined by the ratio of intensities of one variant варианта $\left(J_{1}\right)$ to another $\left(J_{2}\right)$

$$
u_{5}=\frac{J_{1}}{J_{2}}
$$

This ratio is expressed in terms of standard and actual labor costs and is used in many cases as an assessment of the level of organization of construction production. At the same time, the indicators of continuity of work, combining uniformity and their rhythm are considered as limitations.

Less frequently, multiple correlation methods are used to assess the quality of construction production organization. For that, there are selected the result indicator (duration of construction process, labor productivity, profit, etc.) and the organizational, technical, technological factors, with an established quantitative relationship between those factors.

The factors included in the model must meet certain requirements such as the presence of quantitative expression, the existence of a connection between the factor and the resulting indicator, the lack of a close connection between the factors.

The quantitative tightness of the relationship in multiple correlation should be assessed by the multiple correlation coefficient (R). To calculate the total correlation coefficient, it is necessary to determine the pair correlation coefficients of r0i between all factors of xi included in the model and the resulting indicator $y$ and all pair correlation coefficients between the factors. All correlation coefficients are written to a square symmetric matrix.

Multiple correlation coefficient is determined by the formula:

$$
R=\sqrt{1-\frac{D}{D_{11}}}
$$

where $D$ is the determinant of the matrix of pair correlation coefficients;

$D_{11}$ is the determinant of the same matrix with the first row and the first column crossed out, i.e. the determinant of the matrix of pair correlation coefficients between factors.

The simplest form of expression of multiple dependence is a linear dependence of the form:

$$
\tilde{y}=\alpha_{0}+\alpha_{1} x_{1}+\alpha_{2} x_{2}+\ldots+\alpha_{n} x_{n}
$$

where: $\tilde{y}$ is the resulting indicator;

$x_{I}$ are influencing factors; 
$\alpha_{0}$ is a free term of the regression equation;

$\alpha_{1}$ are coefficients with factors.

In cases of absence of the curve shape or a reasonable hypothesis about it, it is avisable to approximate the relation by a polynomial of $n^{\text {th }}$ degree:

$$
\tilde{y}=\alpha_{0}+\alpha_{1} x_{1}+\alpha_{2} x_{2}+\ldots+\alpha_{12} x_{1} x_{2}+\alpha_{13} x_{1} x_{3} \ldots+\alpha_{11} x_{1}^{2}+\alpha_{22} x_{2}^{2}+\ldots \alpha_{111} x_{1}^{3}+\alpha_{222} x_{2}^{3}+\ldots
$$

The degree of a polynomial can be very large and the relations can be not only of the first but also of the second, third, etc. degrees. Products of $x_{i} x_{j}$ type can be considered as the relations. When approximating a polynomial of nth degree, each complex term of the polynomial is replaced by an additional conditional variable in the first degree and the further approximation is carried out as for the linear dependence, i.e.:

$$
\begin{gathered}
Z_{12}=x_{1} x_{2}, Z_{13}=x_{1} x_{3} \ldots, Z_{11}=x_{1}^{2}, Z_{22}=x_{2}^{2}, \ldots \\
\tilde{y}=\alpha_{0}+\alpha_{1} x_{1}+\alpha_{2} x_{2}+\ldots+\alpha_{12} Z_{12}+\alpha_{13} Z_{13} \ldots+\alpha_{11} Z_{11}+\alpha_{22} Z_{22}+\ldots \alpha_{111} Z_{111}+\alpha_{222} Z_{222}
\end{gathered}
$$

The approximation of the multivariate relationship is to identify the regression coefficients for a predetermined form of the regression line. For this purpose, the least squares method is used, the essence of which is that a line is chosen in which the sum of the squares of the differences between the observations and the calculated values will be minimal, i.e.:

$$
S=\Sigma(y-\tilde{y})^{2} \rightarrow \min
$$

where $y, \tilde{y}$, respectively, are the observation data and the calculated values of the variables in regression formula.

With a linear multivariate dependence, this formula will take the following form:

$$
S=\Sigma\left(y-\alpha_{0}-\alpha_{1} x_{1}-\alpha_{2} x_{2}-\ldots-\alpha_{n} x_{n}\right)^{2} \rightarrow \min
$$

\section{Conclusion}

In construction practice, to evaluate organizational decisions, a large variety of different indicators is used: duration and laboriousness of the construction, construction costs and cost price of construction and installation works, level of thread, continuity and intensity of construction of buildings and structures. Some of these indicators are considered to be private or individual, others, on the contrary, are generalizing.

However, organizational decisions made both in design, organizational-technological documentation, and in the process of operational management of the building are based on many technological, technical, organizational, economic, social and other factors and, therefore are multivariate. This means, that the choice of a rational option should be based on an objective and comprehensive assessment.

In order to obtain such estimates, it is recommended to use methods of complex evaluation of solutions, which include methods of criteria assessment, methods of modeling indicators and methods of multiple correlation, each of which has its own application and does not exclude the use of generalizing and particular indicators during the process of option considering.

This work was financially supported by Ministry of Education and Science of the Russian Federation (\#NSh-3492.2018.8). 


\section{References}

1. P.P. Oleinik, T.K. Kuzmina, Industrial and civil engineering, 11, 42-43 (2012)

2. T.K. Kuzmina, Technology and organization of construction production, 1, 50-53 (2012)

3. P.P.Oleinik, T.K. Kuzmina, Technology in textile industry, 3 (369), 11-16 (2017)

4. P.P. Oleinik, V.I. Brodsky, Construction technology and organization of production, 3 (4), 35-38 (2013)

5. T. Kuzmina and N. Cherednichenko, MATEC Web Conf., 86, 05012 (2016)

6. $\quad$ P. Oleinik, T. Kuzmina, V. Zenov, MATEC Web Conf., 86, 05019 (2016)

7. P.P. Oleinik, Natural and technical sciences, 10, 412-414 (2015)

8. L.V. Kievsky, Organizing: from construction process - to investment processes in construction. In edition: Urban development, scientific papers 2006-2014 ed. By L.V. Kievsky (Moscow, 205-221, 2014)

9. P.P. Oleinik, V.I. Brodsky, Technology and organization of construction production, 1(2), 27-31 (2013)

10. P. Oleynik, T. Kuzmina, MATEC Web Conf., 1177, 00129 (2017)

11. L.V.Kievskiy, I.L. Kievskiy, International Journal of Applied Engineering Research, 11(1), 304-311 (2016)

12. Clifford F. Gray, Erik W. Larson, Project management: a practical guide. Translation from English (Moscow, Business and Service Publishing house, 2003)

13. B.F.Shirshikov, A.M. Slavin, Civil and industrial engineering, 8, 92-95 (2016)

14. Frame J. Davidson, The New Project Management: tools for an age of rapid change, complexity, and other business realities. By J. Davidson Frame. $2^{\text {nd }}$ ed. San Francisco: Jossey-bass, A Wiley Company, 360 (2002)

15. Charvat Jason, Project Management Methodologies-Selecting, Implementing, and Supporting Methodologies and Processes for Projects (New Jersey, John Wiley \& Sons inc, 264, 2003) 